\title{
Mean Amplitudes of Vibration in Molecules with Internal Rotation: Halogenated Ethanes
}

\author{
S. J. C Y V I N, I. E L V E B R E D D, B. N. C Y V I N \\ J. BRUNVOLL and G. HAGEN
}

Institutt for fysikalsk kjemi, Norges tekniske høgskole, Trondheim, Norway

\begin{abstract}
Mean amplitudes of vibration calculated from spectroscopic data are reported for hexafluoro-, hexachloro-, and hexabromo ethanes. The framework mean amplitudes of the same molecules are discussed, and calculated for various equilibrium configurations with differing angles of torsion. The resulting variations are found to be not at all negligible. For hexachloroethane taken as example the framework mean amplitudes of the long $\mathrm{Cl}$...Cl distances vary from 0.1336 in the cis position to 0.1156 in gauche, and finally $0.0670 \AA$ in the trans position; all values at $298^{\circ} \mathrm{K}$. A general (approximate) formula is deduced for a variation of this kind, viz.
\end{abstract}

$$
\left\langle\Delta \varrho^{2}\right\rangle \text { frame }=\left(\alpha+\beta \cos \theta+\gamma \cos ^{2} \theta\right) / \varrho^{2}
$$

where $\alpha, \beta$ and $\gamma$ are constants, $\theta$ is the equilibrium angle of torsion, and $\varrho$ is the angle-dependent interatomic distance.

\begin{abstract}
This is the first communication of our spectroscopic studies of molecules 1 with internal rotation. The project has been initiated by the renewed interest of gas electron-diffraction investigations of such molecules, and highly inspired by the discussions at the first Symposium of Electron Diffraction at Oslo in February 1967. It is our purpose to supply some detailed data including mean amplitudes for framework vibrations, obtained from a spectroscopic normal-coordinate analysis of various molecules exhibiting internal rotation. As far as we know such material has been published before only for one molecule, viz. hexachlorodisilane, for which Morino and Hirota ${ }^{1}$ have plotted the long Cl...Cl framework mean amplitude as a function of the torsional angle in different equilibrium positions. The framework mean amplitudes are of prime interest when interpreting the electron-diffraction measurements..$^{2,3}$ One has often resorted to assuming the framework mean amplitudes to be independent of the torsional angle, even in very recent works, 4 but also some empirical functional variations have been proposed..$^{5}$ In this connection it is felt that the spectroscopic data might be well appreciated. The following words from one of the cited papers ${ }^{5}$ is taken as a direct challenge to us:
\end{abstract}


"Another method for separating the contribution from the torsional motion would be to compute the contribution of the frame vibrations to the total amplitude from spectroscopic data and molecular force models." "Such a procedure would be an ideal path to follow if spectral assignments and force models were known without question for the somewhat complicated molecules of interest." (J. Karle ${ }^{5}$ ).

We have chosen some hexahalogeno ethanes as the first compounds to be treated in this project for several reasons. (a) They are not too complicated for a complete spectroscopic analysis. (b) Two of them have already been investigated by modern gas electron diffraction., ${ }^{1,6-8}$ (c) Force field have been derived with good confidence from a recent spectroscopic reinvestigation of the molecules. ${ }^{9}$

\section{FORCE FIELDS}

The present calculations on hexafluoro-, hexachloro-, and hexabromoethane are based on the molecular force fields from Carney et al. ${ }^{9} c f$. Table $\mathrm{V}$ of the cited work. The force constants were subjected to slight refinements in order to be adjusted to fit better the observed fundamentals given in Table IV of Carney et al. ${ }^{9}$ It was comparatively easy to adjust the force constants to fit accurately the observed fundamentals in the nondegenerate species. This is not accessible for the fundamentals of the degenerate vibrations, as long as we adhere to the assumption of identical force constants in the $E_{1 g}$ and $E_{1 u}$ blocks, as also was done by Carney et al. ${ }^{9}$ It is essential to maintain this assumption for the purpose of our further computations. As a whole our refinements of force constants are not to be considered as very substantial, and we do not even find it necessary to report the final set of values.

\section{MEAN AMPLITUDES OF VIBRATION}

The derived force fields (see above) were used to calculate the mean amplitudes of vibration with the results shown in Table 1 . The computations are

Table 1. Mean amplitudes of vibration (with torsion included) for hexafluoro-, hexachloro-, and hexabromo ethanes with staggered equilibrium structures; $\AA$ units. Also included are the framework mean amplitudes for the $X_{1} X_{5}$ (gauche) distances. For all the other

distance types the mean amplitudes are independent of the torsional mode.

\begin{tabular}{|c|c|c|c|c|c|c|}
\hline \multirow{2}{*}{$\mathrm{C}_{2} \mathrm{X}_{6}$} & \multicolumn{2}{|c|}{$\mathrm{C}_{2} \mathrm{~F}_{8}$} & \multicolumn{2}{|c|}{$\mathrm{C}_{2} \mathrm{Cl}_{8}$} & \multicolumn{2}{|c|}{$\mathrm{C}_{2} \mathrm{Br}_{8}$} \\
\hline & $T=0$ & $298^{\circ} \mathrm{K}$ & $T=0$ & $298^{\circ} \mathrm{K}$ & $T=0$ & $298^{\circ} \mathrm{K}$ \\
\hline $\mathrm{C}-\mathrm{C}$ & 0.0507 & 0.0516 & 0.0542 & 0.0551 & 0.0524 & 0.0528 \\
\hline $\mathbf{C}-\mathbf{X}$ & 0.0441 & 0.0446 & 0.0502 & 0.0530 & 0.0498 & 0.0551 \\
\hline C...X & 0.0573 & 0.0647 & 0.0566 & 0.0668 & 0.0538 & 0.0679 \\
\hline $\mathbf{X}_{1} \mathrm{X}_{2}$ & 0.0521 & 0.0556 & 0.0540 & 0.0697 & 0.0468 & 0.0736 \\
\hline $\mathbf{X}_{\mathbf{1}} \mathbf{X}_{\mathbf{1}}$ (trans) & 0.0561 & 0.0625 & 0.0529 & 0.0670 & 0.0445 & 0.0662 \\
\hline $\mathrm{X}_{1} \mathrm{X}_{5}$ (gauche) & 0.0808 & 0.1305 & 0.0773 & 0.1428 & $(0.0632)$ & $(0.1356)$ \\
\hline$X_{1} X_{5}$ (frame) & 0.0720 & 0.0936 & 0.0703 & 0.1156 & 0.0587 & 0.1193 \\
\hline
\end{tabular}


still based on the staggered equilibrium configuration. The torsional mode of vibration contributes (to the first order of approximation) only to the gauche halogen-halogen mean amplitude $\left(\mathrm{X}_{1} \cdots \mathrm{X}_{5}\right)$. The values labeled " $\mathrm{X}_{1} \mathbf{X}_{5}$ (gauche)" in Table 1 are the total mean amplitudes, for which the torsional mode contributions were computed from the frequencies of 68,62 , and $56 \mathrm{~cm}^{-1}$ for $\mathrm{C}_{2} \mathrm{~F}_{6}$, $\mathrm{C}_{2} \mathrm{Cl}_{6}$, and $\mathrm{C}_{2} \mathrm{Br}_{6}$, respectively. The two former values (for $\mathrm{C}_{2} \mathrm{~F}_{6}$ and $\mathrm{C}_{2} \mathrm{Cl}_{6}$ ) are taken from Carney et al., ${ }^{9}$ while the latter (for $\mathrm{C}_{2} \mathrm{Br}_{6}$ ) is an extrapolation. This latter value $\left(56 \mathrm{~cm}^{-1}\right)$ is rather dubious; therefore the mean amplitudes depending on this frequency are given in parentheses in Table 1. For the significance of the values labeled " $\mathrm{X}_{1} \mathrm{X}_{5}$ (frame)" in Table 1 the reader is referred to the discussion below of framework mean amplitudes.

The presently calculated mean amplitudes for $\mathrm{C}_{2} \mathrm{Cl}_{6}$ show satisfactory agreement with the calculated values of Morino and Hirota. ${ }^{1}$ The agreement is rather remarkable in view of the fact that Morino and Hirota used an approximate formula, and based the calculations on an incomplete vibrational assignment of frequencies. For further comparison we have quoted some electron-diffraction values of mean amplitudes for $\mathrm{C}_{2} \mathrm{~F}_{6}$ and $\mathrm{C}_{2} \mathrm{Cl}_{6}$; see Table 2 . They do not show altogether good agreement with our calculated results; the discrepancies may mostly be attributed to relatively large experimental errors in the electron-diffraction experiment.

\section{FRAMEWORK MEAN AMPLITUDES}

Staggered configuration. The concept of framework mean amplitudes was introduced through the theory of electron diffraction,,$^{1-3}$ and is based on an idea of separating the torsional mode from the rest of the normal modes of vibration, which contribute to the mean amplitudes of vibration. As mentioned above the torsional mode in a staggered ethane-type molecule contributes only to the gauche-type $\mathrm{X}$... X mean amplitude. Since the torsional mode

Table 2. Mean amplitudes of vibration ( $\AA$ units) for hexafluoro- and hexachloroethane from electron diffraction.*

\begin{tabular}{|c|c|c|c|c|}
\hline $\mathrm{C}_{2} \mathrm{X}_{6}$ & $\underset{a}{\mathrm{C}_{2} \mathrm{~F}_{6}}$ & $\underset{b}{\mathrm{C}_{2} \mathrm{Cl}_{6}}$ & $\underset{c}{\mathrm{C}_{2} \mathrm{Cl}_{6}}$ & $\underset{d}{\mathrm{C}_{2} \mathrm{Cl}_{6}}$ \\
\hline $\begin{array}{l}\mathrm{C}-\mathrm{C} \\
\mathrm{C}-\mathrm{X} \\
\mathrm{C} \cdots \mathrm{X} \\
\mathbf{X}_{1} \mathbf{X}_{2} \\
\mathbf{X}_{1} \mathbf{X}_{4} \text { (trans) } \\
\mathbf{X}_{1} \mathbf{X}_{5} \text { (gauche) }\end{array}$ & $\begin{array}{l}0.050 \\
0.073 \\
0.057 \\
0.069\end{array}$ & $\begin{array}{r}<0.03 \\
0.03 \\
0.06 \\
0.08\end{array}$ & $\begin{array}{l}0.06_{6} \\
0.06_{8} \\
0.06_{4} \\
0.10_{9} \\
0.13_{6}\end{array}$ & $\begin{array}{l}0.037 \\
0.059 \\
0.069 \\
0.066 \\
0.065 \\
0.101\end{array}$ \\
\hline
\end{tabular}

\footnotetext{
* Limits of error omitted in this table. For detailed accounts the original works $(a-d)$ should be consulted.

a Swick and Karle.?

${ }^{b}$ Swick, Karle and Karle. ${ }^{6}$

$c$ Morino and Hirota. 1

${ }^{d}$ Almenningen, Andersen and Trætteberg. ${ }^{8}$
}

Acta Chem. Scand. 21 (1967) No. 9 
belongs to a symmetry species (viz. $A_{1 u}$ ) all by itself, the separation of normal modes referred to above is easily executed. The framework mean amplitude for the gauche $\mathrm{X}_{1} \cdots \mathrm{X}_{5}$ atom pair is found straightforwardly from the total meansquare amplitude by putting the mean-square amplitude element corresponding to the torsional mode equal to zero, which is equivalent to putting the corresponding force constant and frequency equal to infinity. The values thus obtained for $\mathrm{C}_{2} \mathrm{~F}_{6}, \mathrm{C}_{2} \mathrm{Cl}_{6}$, and $\mathrm{C}_{2} \mathrm{Br}_{6}$ are included in Table 1 under the label " $\mathrm{X}_{1} \mathrm{X}_{5}$ (frame)".

General principles. In the electron-diffraction work one is interested in the framework mean amplitudes as functions of the torsional angle, i.e. for equilibrium structures with differing angles of torsion. The calculation of these quantities implies some assumptions of hypothetic molecules, and the physical significance of the results may seem unclear. Therefore we feel it to be especially important to clarify at least the principles used in the spectroscopic analysis.

$A$ variation of the torsional angle around the complete circle of rotation covers two special structures, viz. the eclipsed structure of symmetry $D_{3 h}$, and staggered of $D_{3 d}$; and intermediate structures of symmetry $D_{3}$. The angle $\theta$ is defined as 0 at the "first eclipsed configuration", i.e. with the atoms numbered 1 and 4 in the cis position, and $180^{\circ}$ at the "second staggered configuration", i.e. with atoms 1 and 4 in the trans position. Through this half rotation the molecule passes a "first staggered configuration" at $\theta=60^{\circ}$ and a "second eclipsed configuration" at $120^{\circ}$.

The basic ideas of the spectroscopic calculations of framework mean amplitudes are (a) the neglecting of the torsional vibrations, and (b) assuming the same force constants for all the molecular configurations throughout the circle of rotation. The last requirement (b) may meet with difficulties of a principal theoretic nature. It may happen, namely, that a tentative application of the force field for the various models comes into conflict with basic symmetry requirements for the force constants. The difficulties may usually be circumvented by assuming a sufficient number of interaction force constants equal to zero as approximations. As a matter of fact the sum rule ${ }^{10,11}$ and product rule ${ }^{11-13}$ for frequencies of rotational isomers have been derived along the same principles, and have been verified experimentally for molecules of which several stable rotational isomers are known.11

In the presently considered types of molecules we must take into consideration the symmetry species of the groups $D_{3 h}$ and $D_{3 d}$, among which the normal frequencies of the eclipsed and staggered molecules are distributed. Fortunately the correlations between these symmetry species allow the application of the same force field to both models. One has only to make the reasonable assumption that the symmetry coordinates from different models, but with formally equal algebraic expressions in terms of the valence coordinates are associated with the same force constant. In particular the torsional mode in the eclipsed model belongs to one species $\left(A_{1}^{\prime \prime}\right)$ all by itself, as also is the case for the staggered model (see above).

The force constants of the eclipsed and staggered models are immediately applicable to any of the intermediate models of symmetry $D_{3}$, and involve the following assumptions. (i) The three interaction force constants involving 
the torsional coordinate in the totally symmetric species $\left(A_{1}\right)$ vanish. (ii) Nine interaction force constants within the double degenerate species $(E)$ vanish. They are the constants combining a symmetric and antisymmetric coordinate (stretching or bending) defined with respect to the two movable $\mathrm{CX}_{3}$ atom groups. By virtue of the assumption (i) the torsional mode is completely separated from the others, since the corresponding coupling terms in the $\mathrm{G}$ matrix are all zero. Hence there is no difficulty in executing the neglect of torsional vibrations; $c f$. (a) above, in order to produce the framework mean amplitudes.

The readers who are interested in further details of the spectroscopic analysis, are referred to another paper ${ }^{14}$ containing a theoretic treatment of the vibrations of the inherent models. That work contains a specification of suitable sets of symmetry coordinates, the correlation between symmetry species of the appropriate symmetry groups, and $G$ matrix elements, along with additional algebraic expressions. The dependence of $G$ matrix elements on the equilibrium angle of torsion is a matter of particular relevance to the present problems.

Results. The force fields from the staggered configurations of hexafluoro-, hexachloro-, and hexabromoethane (see above) were used to calculate the framework mean amplitudes according to the principles outlined in the preceding section. Under the adopted assumptions the framework mean amplitude for the CC distance is strictly the only one being independent of the angle $\theta$ with exactness. But also the variations in the mean amplitudes of bonded $\mathrm{CX}$, nonbonded $\mathrm{C} \cdots \mathrm{X}$, and $1,1-(\mathrm{X} \cdots \mathrm{X})$ are negligible; the variations are beyond the number of decimals here reported (cf. Table 1). The functional
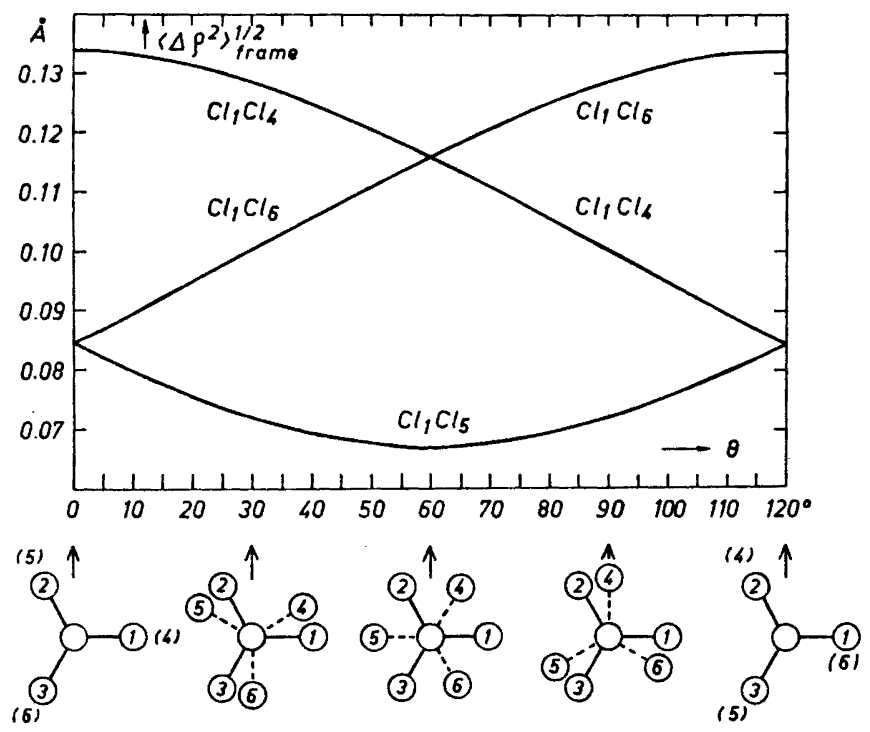

Fig. 1. Framework $\mathrm{Cl}$...Cl mean amplitudes for hexachloroethane at $298^{\circ} \mathrm{K}$ in equilibrium configurations with different angles of torsion, $\theta$.

Acta Chem. Scand. 21 (1967) No. 9 
Table 3. Framework mean amplitudes of vibration for the $\mathrm{X}_{1} \mathrm{X}_{4}$ distance in hexafluoro-, hexachloro-, and hexabromo ethanes with various equilibrium structures; $\AA$ units. $\theta$ is the equilibrium angle of torsion. $T=298^{\circ} \mathrm{K}$.

\begin{tabular}{cccc}
\hline $\begin{array}{c}\mathrm{C}_{2} \mathrm{X}_{6} \\
\theta \text { (degrees) }\end{array}$ & $\mathrm{C}_{2} \mathrm{~F}_{6}$ & $\mathrm{C}_{2} \mathrm{Cl}_{6}$ & $\mathrm{C}_{2} \mathrm{Br}_{6}$ \\
\hline 0 (cis) & 0.1095 & 0.1336 & \\
10 & 0.1089 & 0.1330 & 0.1405 \\
20 & 0.1073 & 0.1312 & 0.1397 \\
30 & 0.1047 & 0.1284 & 0.1375 \\
40 & 0.1014 & 0.1247 & 0.1342 \\
50 & 0.0976 & 0.1204 & 0.1298 \\
60 (gauche) & 0.0936 & 0.1156 & 0.1248 \\
70 & 0.0894 & 0.1105 & 0.1193 \\
80 & 0.0852 & 0.1052 & 0.1136 \\
90 & 0.0813 & 0.0998 & 0.1077 \\
100 & 0.0776 & 0.0944 & 0.1018 \\
110 & 0.0742 & 0.0892 & 0.0959 \\
120 & 0.0712 & 0.0842 & 0.0902 \\
130 & 0.0686 & 0.0795 & 0.0848 \\
140 & 0.0664 & 0.0753 & 0.0797 \\
150 & 0.0647 & 0.0718 & 0.0753 \\
160 & 0.0635 & 0.0692 & 0.0715 \\
170 & 0.0628 & 0.0675 & 0.0686 \\
180 (trans) & 0.0625 & 0.0670 & 0.0669 \\
\hline
\end{tabular}

dependence of $\theta$ for the long $\mathrm{X} \cdots \mathrm{X}$ distances on the other hand is found to be substantial, as is seen from Table 3 and Fig. 1, showing the calculated quantities at $298^{\circ} \mathrm{K}$. In Fig. 1 the long $\mathrm{Cl}$... Cl framework mean amplitudes for $\mathrm{C}_{2} \mathrm{Cl}_{6}$ are represented graphically. In fact the three curves for $\mathrm{Cl}_{1} \mathrm{Cl}_{4}, \mathrm{Cl}_{1} \mathrm{Cl}_{5}$, and $\mathrm{Cl}_{1} \mathrm{Cl}_{6}$ between $\theta=0$ and $60^{\circ}$ would be sufficient to specify all the types of long $\mathrm{Cl}$...Cl distances, but the curves have been extended until $\theta=120^{\circ}$ for the sake of clarity. All the values could alternatively be represented by using only the $\mathrm{Cl}_{1} \mathrm{Cl}_{4}$ distance; then the range of torsional angles would have to be extended from $\theta=0$ until $180^{\circ}$. This last principle is the one utilized in setting up Table 3 .

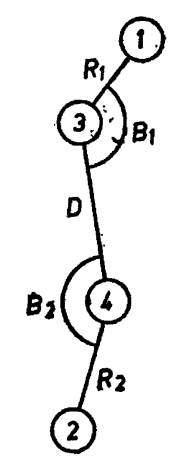

Fig. 2. Notation used for equilibrium parameters in a $1-3-4-2$ chain of atoms in a molecule. 


\section{APPROXIMATE FORMULA}

General formulation. Consider the 1-2 distance between the end atoms in a 1-3-4-2 chain like that shown in Fig. 2. For this distance, $\varrho$, one has

$$
\begin{aligned}
\varrho^{2}(\theta)=R_{1}^{2} & +R_{2}^{2}+2 R_{1} R_{2}\left(\cos B_{1} \cos B_{2}-\sin B_{1} \sin B_{2} \cos \theta\right) \\
& +D^{2}-2 R_{1} D \cos B_{1}-2 R_{2} D \cos B_{2}
\end{aligned}
$$

where $R_{1}, R_{2}, D, B_{1}$, and $B_{2}$ are the structural parameters explained on Fig. 2, and $\theta$ is the angle of torsion between the $1-3-4$ and $2-4-3$ planes. Under the assumption of small vibrations usually made in the spectroscopic analysis of polyatomic molecules, one has for the 1-2 distance deviations

$$
\Delta \varrho=\sum_{i=1,2}\left(\frac{\partial \Delta \varrho}{\partial \Delta R_{i}} \Delta R_{i}+\frac{\partial \Delta \varrho}{\partial\left(R_{i} \Delta B_{i}\right)} R_{i} \Delta B_{i}\right)+\frac{\partial \Delta \varrho}{\partial \Delta D} \Delta D+\frac{\partial \Delta \varrho}{\partial \Delta \theta} \Delta \theta
$$

Here the partial derivatives are supposed to be taken at the equilibrium configuration, and consequently

$$
\begin{aligned}
& \varrho \partial \Delta \varrho / \partial \Delta R_{i}=R_{i}-D \cos B_{i}+R_{j}\left(\cos B_{i} \cos B_{j}-\sin B_{i} \sin B_{j} \cos \theta\right) \\
& \varrho \partial \Delta \varrho / \partial\left(R_{i} \Delta B_{i}\right)=D \sin B_{i}-R_{j}\left(\sin B_{i} \cos B_{j}+\cos B_{i} \sin B_{j} \cos \theta\right) ; \\
& \varrho \partial \Delta \varrho / \partial \Delta D=D-R_{1} \cos B_{1}-R_{2} \cos B_{2} \\
& \varrho \partial \Delta \varrho / \partial \Delta \theta=R_{1} R_{2} \sin B_{1} \sin B_{2} \sin \theta
\end{aligned}
$$

From eqns. (2) and (3) we may form the mean-square amplitude of $\left\langle\Delta \varrho^{2}\right\rangle$. In order to obtain the pertinent framework mean-square amplitude we put $\left\langle\Delta \theta^{2}\right\rangle=0$, and obtain a result of the form

$$
\varrho^{2}\left\langle\Delta \varrho^{2}\right\rangle_{\mathrm{fr}}=\alpha+\beta \cos \theta+\gamma \cos ^{2} \theta
$$

Here the coefficients of $\alpha, \beta$, and $\gamma$ are composed of the various mean-square amplitude quantities, viz. $\left\langle\Delta R_{i}{ }^{2}\right\rangle,\left\langle\Delta B_{i}{ }^{2}\right\rangle(i=1,2)$, and $\left\langle\Delta D^{2}\right\rangle$, and also interaction terms of the types $\left\langle\Delta R_{1} \Delta R_{2}\right\rangle,\left\langle\Delta R_{1} \Delta B_{i}\right\rangle,\left\langle\Delta R_{1} \Delta D\right\rangle$, etc. The terms of $\left\langle\Delta R_{i}{ }^{2}\right\rangle$ and $\left\langle\Delta D^{2}\right\rangle$ are recognized as the mean-square amplitudes of vibration for the bonded distances within the $1-3-4-2$ chain. Also the nonbonded $1 \cdots 4$ and $2 \cdots 3$ mean-square amplitudes, say $\left\langle\Delta S_{1}^{2}\right\rangle$ and $\left\langle\Delta S_{2}^{2}\right\rangle$ may be evaluated in terms of the above mentioned quantities. They may be deduced from the formula

$$
\begin{aligned}
S_{i} \Delta S_{i} & =\left(R_{i}-D \cos B_{i}\right) \Delta R_{i}+\left(D \sin B_{i}\right) R_{i} \Delta B_{i} \\
& +\left(D-R_{i} \cos B_{i}\right) \Delta D ; \quad i=1,2
\end{aligned}
$$

where $S_{i}$ represents the equilibrium distances of the nonbonded atoms in question.

So far the analysis is quite general, as it applies to any four atoms forming a chain like shown in Fig. 2 as part of a molecule. But the mean-square amplitude quantities depend in various ways upon the mean-square values of normal coordinates, having different properties according to the symmetries of the molecular models in question.

In practical applications of eqn. (4) it seems reasonable to regard the $\alpha$, $\beta$, and $\gamma$ coefficients as constant parameters, i.e. independent of the angle $\theta$.

Acta Chem. Scand. 21 (1967) No. 9 
According to the above discussion this implies some assumptions about constancies of the inherent mean-square amplitude quantities.

Application to the hexahalogeno ethanes. Eqn. (4) was applied to the presently treated $\mathrm{C}_{2} \mathrm{X}_{6}$ molecules. Hereby we regarded $\alpha, \beta$, and $\gamma$ as empirical constants, which we computed by means of three "known" values taken from the rigorous calculations; Table 3 . Incidently we used the values at $\theta=0$, $90^{\circ}$, and $180^{\circ}$. The framework mean-square amplitudes thus calculated showed a perfect agreement with those from the rigorous calculations. We even hesitated to refer to eqn. (4) as an "approximate" formula. At least for the presently considered $\mathrm{C}_{2} \mathrm{X}_{6}$ molecular models it is clear that the $\alpha, \beta$, and $\gamma$ coefficients of eqn. (4) are (almost) independent of $\theta$ with a high degree of accuracy under the presently assumed conditions.

Some attempts were made to explain the apparent constancy of the $\alpha$, $\beta$, and $\gamma$ coefficients in the cases of the present calculations. The following properties of the inherent mean-square amplitude quantities were deduced under the present assumptions about the force fields.

(i) $\left\langle\Delta D^{2}\right\rangle,\left\langle\Delta R_{i} \Delta D\right\rangle$, and $\left\langle\Delta B_{i} \Delta D\right\rangle(i=1,2)$ are entirely independent of $\theta$.

(ii) $\left\langle\Delta R_{i}^{2}\right\rangle$ has the same value for $\theta=0,60^{\circ}, 120^{\circ}$, and $180^{\circ}$; and the same is true for $\left\langle\Delta B_{i}{ }^{2}\right\rangle$ and $\left\langle\Delta R_{i} \Delta B_{i}\right\rangle$. The proof is outlined below for the case of $\left\langle\Delta R_{i}^{2}\right\rangle$. Using the notation of Ref. 14 , it is to be proved that $\left\langle r^{2}\right\rangle$ for the eclipsed and staggered models are identical. The quantities may be evaluated in terms of the mean-square amplitude matrix elements as follows.

$$
\begin{aligned}
& \left\langle r^{2}\right\rangle_{\text {eclipsed }}=\frac{1}{6}\left[\Sigma_{1}\left(A_{1}{ }^{\prime}\right)+\Sigma_{1}\left(A_{2}{ }^{\prime \prime}\right)\right]+\frac{1}{3}\left[\left(\Sigma_{1}\left(E^{\prime}\right)+\Sigma_{1}\left(E^{\prime \prime}\right)\right]\right. \\
& \left\langle r^{2}\right\rangle_{\text {staggered }}=\frac{1}{6}\left[\left(\Sigma_{1}\left(A_{1 g}\right)+\Sigma_{1}\left(A_{2 u}\right)\right]+\frac{1}{3}\left[\Sigma_{1}\left(E_{g}\right)+\Sigma_{1}\left(E_{u}\right)\right]\right.
\end{aligned}
$$

The two first terms of these evaluations are the same because the $\Sigma$ matrix elements of the $A_{1}{ }^{\prime}$ and $A_{2}{ }^{\prime \prime}$ species are identical with those of $A_{2 g}$ and $A_{2 u}$, respectively. This is a consequence of identical $\mathrm{G}$ matrix elements, ${ }^{14}$ and our choice of same force constants. For the degenerate species one has detected a "switched" correspondence, i.e. $\mathrm{G}\left(E^{\prime}\right)=\mathrm{G}\left(E_{u}\right)$ and $\mathrm{G}\left(E^{\prime \prime}\right)=\mathrm{G}\left(E_{\mathrm{g}}\right)$; and consequently $\Sigma\left(E^{\prime}\right)=\Sigma\left(E_{u}\right)$ and $\Sigma\left(E^{\prime \prime}\right)=\Sigma\left(E_{g}\right)$. Hence eqns. (6) and (7) give entirely identical results. For intermediate positions (symmetry $D_{3}$ ) the corresponding equation reads

$$
\left\langle r^{2}\right\rangle=\frac{1}{6}\left[\Sigma_{1}\left(A_{1}\right)+\Sigma_{1}\left(A_{2}\right)\right]+\frac{1}{3}\left[\Sigma_{1}(E)+\Sigma_{4}(E)\right]
$$

One expects of course the results from this equation (for all values of $\theta$ ) to come very near the result from eqn. (6) or (7). We actually obtained complete numerical equivalence, but have not been able to prove or disprove an exact equivalence under the assumed conditions.

(iii) The interaction terms $\left\langle\Delta R_{1} \Delta R_{2}\right\rangle,\left\langle\Delta B_{1} \Delta B_{2}\right\rangle$, and $\left\langle\Delta R_{1} \Delta B_{2}\right\rangle$ do vary with the angle $\theta$, but the quantities are very small. To take the stretching coordinates for illustration, we give the expressions for $\left\langle r_{1} r_{4}\right\rangle$ (in the notation of Ref. 14). For the eclipsed and staggered models:

$$
\begin{aligned}
& \left\langle r_{1} r_{4}\right\rangle_{\text {eclipsed }}=\frac{1}{6}\left[\Sigma_{1}\left[A_{1}{ }^{\prime}\right)-\Sigma_{1}\left(A_{2}{ }^{\prime \prime}\right)\right]+\frac{1}{3}\left[\Sigma_{1}\left(E^{\prime}\right)-\Sigma_{1}\left(E^{\prime \prime}\right)\right] \\
& \left\langle r_{1} r_{4}\right\rangle_{\text {staggered }}=\frac{1}{6}\left[\Sigma_{1}\left(A_{1 g}\right)-\Sigma_{1}\left(A_{2 u}\right)\right]+\frac{1}{3}\left[\Sigma_{1}\left(E_{g}\right)-\Sigma_{1}\left(E_{u}\right)\right]
\end{aligned}
$$

For intermediate positions the appropriate formula reads

$$
\left\langle r_{1} r_{4}\right\rangle=\frac{1}{6}\left[\Sigma_{1}\left(A_{1}\right)-\Sigma_{1}\left(A_{2}\right)\right]+\frac{1}{3}\left[\Sigma_{1}(E)-\Sigma_{4}(E)\right]
$$


Table 4. Applied equilibrium distances $(\AA)$ for $\mathrm{C}_{2} \mathrm{~F}_{6}, \mathrm{C}_{2} \mathrm{Cl}_{6}$, and $\mathrm{C}_{2} \mathrm{Br}_{6}$; and the coefficients of eqn. (4) at $298^{\circ} \mathrm{K}$ ( $\AA^{4}$ units).

\begin{tabular}{|c|c|c|c|c|c|}
\hline \multirow[t]{2}{*}{$\mathrm{C}_{2} \mathrm{X}_{6}$} & \multirow[t]{2}{*}{$C-X$} & \multirow[t]{2}{*}{$\mathrm{C}-\mathrm{C}$} & \multicolumn{3}{|c|}{ Coefficients of eqn. (4) } \\
\hline & & & $\alpha$ & $\boldsymbol{\beta}$ & $\gamma$ \\
\hline $\mathrm{C}_{2} \mathrm{~F}_{6}$ & 1.32 & 1.56 & 0.0596 & 0.01194 & -0.00019 \\
\hline $\mathrm{C}_{2} \mathrm{Cl}_{8}$ & 1.76 & 1.54 & 0.1176 & 0.02454 & -0.01065 \\
\hline $\mathrm{C}_{2} \mathrm{Br}_{6}$ & 1.93 & 1.52 & 0.1371 & 0.03136 & -0.01310 \\
\hline
\end{tabular}

For the sake of illustration we have evaluated numerically the $\left\langle r_{1} r_{4}\right\rangle$ terms for $\mathrm{C}_{2} \mathrm{Cl}_{6}$ in various equilibrium positions at $298^{\circ} \mathrm{K}$. The magnitudes run from $0.0000251 \AA^{2}$ in the cis position of the $1-4$ atoms to $-0.0000002 \AA^{2}$ in trans.

Results. Table 4 shows the coefficients of eqn. (4) for the hexahalogeno ethanes from the present calculations at $298^{\circ} \mathrm{K}$. Also included are the adopted interatomic distances ${ }^{9}$ used as equilibrium parameters throughout the computations of the present work. All equilibrium interbond angles were assumed to be tetrahedral $\left(109.471^{\circ}\right)$. As already mentioned the application of eqn. (4) reproduced the results of the rigorous calculations (Table 3) with practically numerical exactness.

\section{EMPIRIC FORMULAE}

Comparison with Karle's formula. Karle ${ }^{5}$ has suggested a formula for the angle-dependence of framework mean-square amplitudes, which (adapted to our notation) reads

$$
\left\langle\Delta \varrho^{2}\right\rangle_{\mathrm{fr}}=a\left[1+b \exp \left(-\theta^{2}\right)\right] ; \quad 0 \leq \theta \leq \pi
$$

It was not possible to adjust this two-parameter formula to our data with any reasonable tolerance. We therefore tentatively adjusted the formula to the three "known" values at $\theta=0, \pi / 2$, and $\pi$ according to

Fig. 3. Framework mean-square $\mathrm{Cl} \ldots \mathrm{Cl}$ amplitudes for hexachloroethane at $298^{\circ} \mathrm{K}$ in equilibrium configurations with different angles of torsion, $\theta$.

(I) Eqn. (4) with the coefficients given in Table 4. The curve is practically coincident with the curve from rigorous calculations (cf. Table 3).

(II) Eqn. (13) with (a) $a^{\prime}=0.009252$, $b^{\prime}=0.9299$, (b) $a^{\prime \prime}=0.004480, b^{\prime \prime}=14.477$. (III) Eqn. (14) with $a=0.003243$, $b=4.5055$.

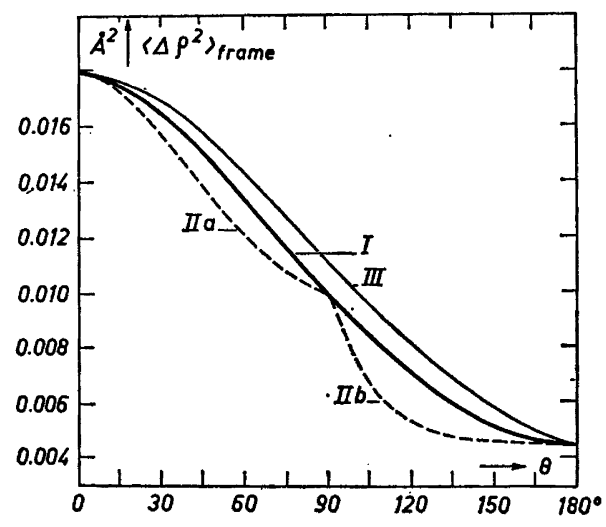

Acta Chem. Scand. 21 (1967) No. 9 


$$
\begin{aligned}
& \left\langle\Delta \varrho^{2}\right\rangle_{\mathrm{fr}}=a^{\prime}\left[1+b^{\prime} \exp \left(-\theta^{2}\right)\right] ; \quad 0 \leq \theta \leq(\pi / 2) \\
& \left\langle\Delta \varrho^{2}\right\rangle_{\mathrm{fr}}=a^{\prime \prime}\left[1+b^{\prime \prime} \exp \left(-\theta^{2}\right)\right] ; \quad(\pi / 2) \leq \theta \leq \pi
\end{aligned}
$$

Eqns. (13) virtually represent a three-parameter function, similarly to our eqn. (4). The results from eqns. (13) are included in the diagram of Fig. 3, and show rather unsatisfactory fit to the curve from rigorous calculations. Some of the largest discrepancies are found for $\theta=60^{\circ}$ (in branch IIa of Fig. 3), viz. $-9.3 \%$; and for $\theta=120^{\circ}$ (in branch IIb) $-25.3 \%$.

Modified Karle's formula. It was found that a slight modification of eqn. (12), viz.

$$
\left\langle\Delta \varrho^{2}\right\rangle_{\mathrm{fr}}=a\left[1+b \exp \left(-\theta^{2} / 4\right)\right] ; \quad 0 \leq \theta \leq \pi
$$

gave a qualitatively good reproduction of the rigorously calculated results throughout the range of $\theta$ values, when the two parameters $a$ and $b$ were properly adjusted. The corresponding curve is shown in Fig. 3 (branch III), and displays a deviation of $+11.5 \%$ from the rigorously calculated value at $\theta=90^{\circ}$.

Possibility of estimating function parameters. We turn back to the function of eqn. (4) for some final comments. The superiority of this formula as compared to other functional dependences seems now to have been clearly demonstrated. The only serious obstacle against eqn. (4) is the presence of three adjustable parameters, which may not be accessible in cases where the complete spectroscopic analysis is not available. For this reason it might be worth while making further efforts to correlate these parameters to the mean-square amplitude quantities, of which they are composed. The attempts we have made so far were mainly unsuccessful, and we do not intend to pursue the matter further at the present moment. It seems more profitable to continue these studies by an application of eqn. (4) to some simpler molecules, such as $\mathrm{S}_{2} \mathrm{Cl}_{2}$ and perhaps $\mathrm{B}_{2} \mathrm{Cl}_{4}$.

\section{REFERENCES}

1. Morino, Y. and Hirota, E. J. Chem. Phys. 28 (1958) 185.

2. Karle, J. and Hauptmann, H. J. Chem. Phys. 18 (1950) 875.

3. Karle, J. J. Chem. Phys. 22 (1954) 1246.

4. Knudsen, R. E., George, C. F. and Karle, J. J. Chem. Phys. 44 (1966) 2334.

5. Karle, J. J. Chem. Phys. 45 (1966) 4149.

6. Swick, D. A., Karle, I. L. and Karle, J. J. Chem. Phys. 22 (1954) 1242.

7. Swick, D. A. and Karle, I. L. J. Chem. Phys. 23 (1955) 1499.

8. Almenningen, A., Andersen, B. and Trætteberg, M. Acta Chem. Scand. 18 (1964) 603.

9. Carney, R. A., Piotrowski, A., Meister, A. G., Braun, J. H. and Cleveland, F. F. J. Mol. Spectry. 7 (1961) 209.

10. Mizushima, S., Shimanouchi, T., Nakagawa, I. and Miyake, A. J. Chem. Phys. 21 (1953) 215.

11. Mizushima, S. Structure of Molecules and Internal Rotation, Academic, Now York 1954.

12. Mizushima, S., Morino, Y. and Shimanouchi, T. Sci. Papers Inst. Phys. Chem. Res. (Tokyo) 40 (1942) 87.

13. Bernstein, H. J. J. Chem. Phys. 17 (1949) 256.

14. Cyvin, S. J., Elvebredd, I., Brunvoll, J. and Hagen, G. To be published.

Received May 15, 1967.

Acta Chem. Scand. 21 (1967) No. 9 